\title{
Tetrabromobisphenol-A/S and Nine Novel Analogs in Biological Samples from the Chinese Bohai Sea: Implications for Trophic Transfer
}

\author{
Ai-feng Liu, ${ }^{\dagger}$ Guang-bo Qu, ${ }^{\dagger}$ Miao Yu, ${ }^{\dagger}$ Yan-wei Liu, ${ }^{\dagger}$ Jian-bo Shi, ${ }^{*},+$, and Gui-bin Jiang ${ }^{\dagger, \S}$ \\ ${ }^{\dagger}$ State Key Laboratory of Environmental Chemistry and Ecotoxicology, Research Center for Eco-Environmental Sciences, Chinese \\ Academy of Sciences, P.O. Box 2871, Beijing 100085, China \\ ${ }^{\ddagger}$ Institute of Environment and Health, Jianghan University, Wuhan 430056, China \\ ${ }^{\S}$ College of Resources and Environment, University of Chinese Academy of Sciences, Beijing 100049, China
}

Supporting Information

\begin{abstract}
Tetrabromobisphenol-A/S (TBBPA/S) analogs have raised substantial concern because of their adverse effects and potential bioaccumulative properties, such as TBBPA bis(allyl ether) (TBBPA-BAE) and TBBPA bis(2,3-dibromopropyl ether) (TBBPA-BDBPE). In this study, a comprehensive method for simultaneous determination of TBBPA/S and nine novel analogs, including TBBPA-BAE, TBBPA-BDBPE, TBBPS-BDBPE, TBBPA mono(allyl ether) (TBBPA-MAE), TBBPA mono(2-bromoallyl ether) (TBBPA-MBAE), TBBPA mono(2,3-dibromopropyl ether) (TBBPA-MDBPE), TBBPSMAE, TBBPS-MBAE, and TBBPS-MDBPE in biological samples was developed. The distribution patterns and trophic transfer properties of TBBPA/S and analogs in various biological samples collected from the Chinese Bohai Sea were then studied in detail. For the first time, TBBPA-MBAE and TBBPS-BDBPE were detected in biological samples and TBBPAMBAE was identified as a byproduct. The concentrations of TBBPA and analogs ranged from ND (not detected or below the method detection limit) to $2782.8 \mathrm{ng} / \mathrm{g}$ lipid weight (lw), and for TBBPS and analogs ranged from ND to $927.8 \mathrm{ng} / \mathrm{g}$ lw. High detection frequencies (>86\%) for TBBPA, TBBPS and TBBPA-MAE, TBBPA-MDBPE, TBBPS-MAE, TBBPS-MBAE, and TBBPS-MDBPE were obtained. Meanwhile, TBBPA, TBBPS, and these five analogs displayed trophic dilution tendencies due to significantly negative correlations between trophic levels and lipid-corrected concentrations together with the trophic magnification factors (from 0.31 to 0.55 ). The results also indicated the novel TBBPA-MAE, TBBPA-MBAE, TBBPA-MDBPE, TBBPS-MAE, TBBPS-MBAE, and TBBPS-MDBPE could be generated not only as byproducts, but also as the probable transformation products of commercial TBBPA/S derivatives.
\end{abstract}

\section{INTRODUCTION}

Brominated flame retardants (BFRs) are the subject of widespread concern due to their extensive use and potential environmental and health risks. ${ }^{1,2}$ Tetrabromobisphenol-A (TBBPA) is one of the most important BFRs, and represents about $60 \%$ of the total BFR market. TBBPA and commercially available analogs, such as tetrabromobisphenol-S (TBBPS), TBBPA bis(allyl ether) (TBBPA-BAE), TBBPA bis(2,3dibromopropyl ether) (TBBPA-BDBPE), and TBBPS bis(2,3dibromopropyl ether) (TBBPS-BDBPE), are used as additive and reactive BFRs in print circuit boards, plastics, etc. ${ }^{1}$ The EU risk assessment confirmed the environmental risk of using TBBPA as an additive in BFRs due to their increased leakage potential. ${ }^{3}$ TBBPA-BAE, TBBPA-BDBPE, and TBBPS$\mathrm{BDBPE}^{1,2}$ have been found in soil, dust, sewage sludge, and eggs, with the highest observed concentration being $9960 \mathrm{ng} / \mathrm{g}$ dry weight $(\mathrm{dw}) .^{4-8}$ Since TBBPA has been extensively evaluated for its endocrine disruption effects, neurotoxicity and reproductive-development toxicity, the recently reported toxic assessments for TBBPA derivatives mainly focused on the potential neurotoxic effects of TBBPA-BAE, ${ }^{9}$ maternal transfer properties of TBBPA-BDBPE ${ }^{10}$ and the slow liver elimination rate of TBBPA-BDBPE in rats. ${ }^{11}$

The wide application of TBBPA/S in consumer products has resulted in the occurrence of byproducts or degradation products in the environment as emerging contaminants. ${ }^{12,13}$ Several byproducts of TBBPA-BAE, TBBPA-BDBPE, and TBBPS-BDBPE have been identified as novel contaminants and these byproducts have been shown to pose more severe

Received: December 31, 2015

Revised: $\quad$ March 8, 2016

Accepted: March 23, 2016

Published: March 23, 2016 
Table 1. Structures and Physical-Chemical Properties of TBBPA/S and Nine Analogs

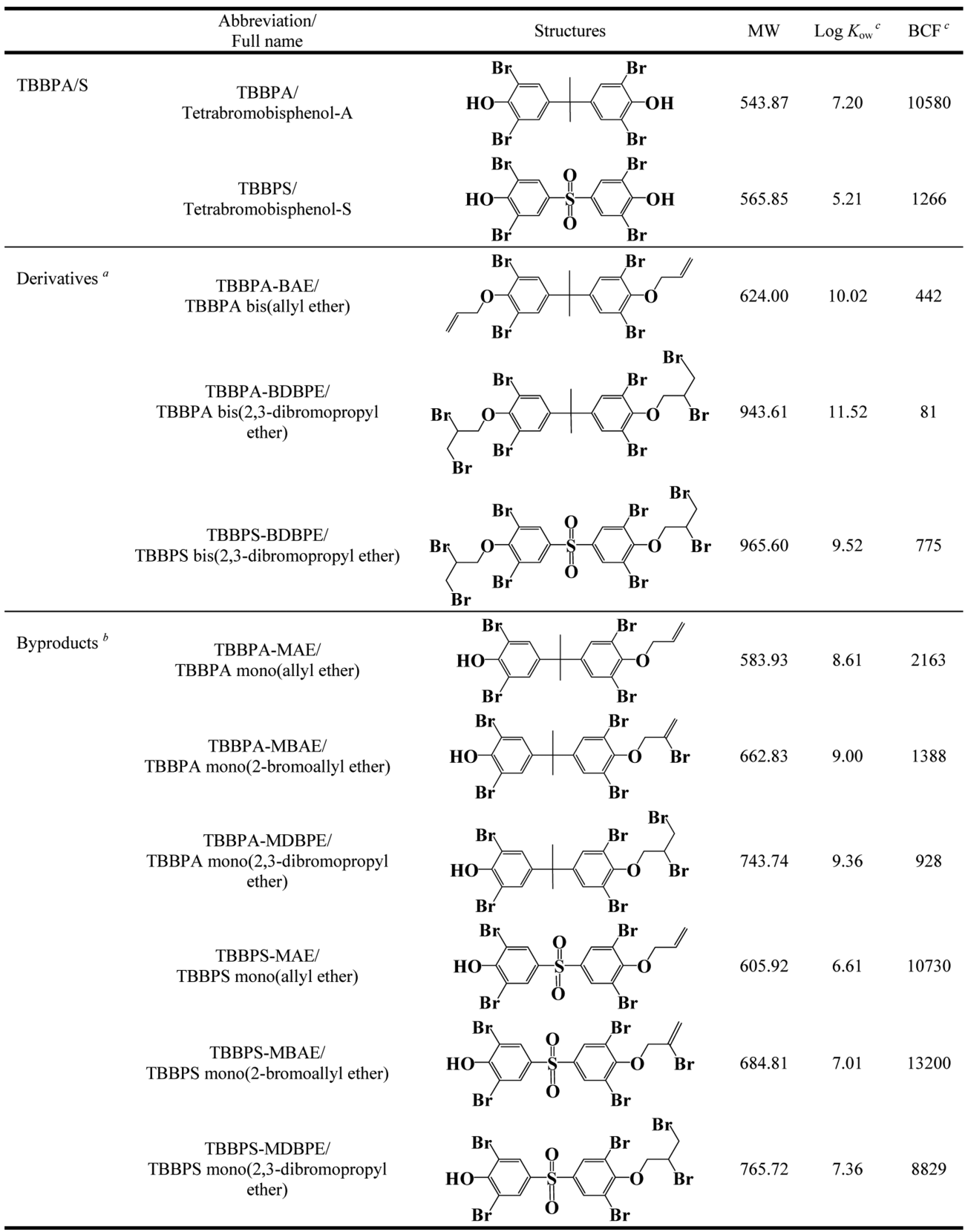

${ }^{a}$ The derivatives are produced in the BFR factories in large amounts and they are synthesized from modification of two phenol groups of TBBPA/S. ${ }^{b}$ The byproducts are not commercially available, and they are produced with the derivatives as impurities. ${ }^{c}$ Calculated with U.S. EPA EPI V4.1.

environmental risks than TBBPA-BAE, TBBPA-BDBPE, and TBBPS-BDBPE, such as TBBPA mono(allyl ether) (TBBPAMAE), TBBPA mono(2,3-dibromopropyl ether) (TBBPAMDBPE), TBBPS mono(allyl ether) (TBBPS-MAE), TBBPS mono(2-bromoallyl ether) (TBBPS-MBAE), and TBBPS mono(2,3-dibromopropyl ether) (TBBPS-MDBPE). ${ }^{13-15}$ TBBPA-BAE, TBBPA-BDBPE, and TBBPS-BDBPE are linked by the $\mathrm{C}($ aryl $)-\mathrm{O}-\mathrm{C}($ alkyl $)$ bond, and cleavage of the $\mathrm{O}-$ $\mathrm{C}$ (alkyl) bond is quite sensitive to the bacterial biodegradation, UV irradiation and super-reduced conditions. ${ }^{16-18}$ TBBPABDBPE has been observed to transform into TBBPA through hydrolysis in aquatic mesocosms. ${ }^{19}$ TBBPA/S derivatives have the potential for ether bond cleavage and formation of the monomodified degradation products or byproducts. ${ }^{13,14}$ Based on their physical-chemical properities and primary toxicities, these byproducts or degradation products have been predicted to be more bioaccumulative and toxic than TBBPA/S derivatives. $^{15,20}$

Dietary intake, especially seafood consumption, is a major pathway of human exposure to some BFRs, metabolites of which have also been found in marine organisms and human breast milk. $^{20-22}$ Currently, TBBPA/S and analogs have mainly been analyzed in abiotic matrices and river organisms, and only a few studies have been conducted in marine environments. ${ }^{22}$ Although its concentration is low, TBBPA has been detected in different marine organisms including mollusks, crab, fish, and porpoises collected from the North Sea. ${ }^{23}$ The novel TBBPA/S analogs, TBBPA-MAE, TBBPA-MDBPE, TBBPS-MAE, TBBPS-MBAE, and TBBPS-MDBPE were identified in mollusk samples with concentrations ranging from ND (not detected or 
below the method detection limit) to $4.1 \mathrm{ng} / \mathrm{g} \mathrm{dw}{ }^{14,15}$ However, the bioaccumulation and trophic transfer properties of these novel TBBPA/S analogs in the aquatic food web have never been studied and much about them remains unknown. Moreover, further study of novel TBBPA/S analogs in complex biota matrices has also been impeded by a lack of available methods and pure standards.

Various methods have been developed for the detection of TBBPA/S and their derivatives. TBBPA-BDBPE was first detected using a high pressure liquid chromatography-diode array detector (HPLC-DAD); in this research, the high detection limit revealed the need for more sensitive method. ${ }^{24}$ Gas chromatography coupled with mass spectrometry (GCMS) was also applied in the analysis of TBBPA-BDBPE; ${ }^{6}$ however, this method was controversial because of its farfetched mass spectrum explanation. ${ }^{5}$ The thermolability could induce the decomposition of TBBPA/S and their derivatives in the process of programming temperature increase in GC-MS. ${ }^{1,2}$ Recently developed reactive extractive electrospray ionization tandem mass spectrometry (EESI-MS) provided an alternative approach for sensitive analysis of TBBPA-BAE, but this method was only applied for simple matrix analysis, such as water samples. ${ }^{25,26}$ Atmospheric pressure photoionization mass spectrometry (APPI-MS) was sensitive and specific for TBBPA-BAE, TBBPA-BDBPE, and TBBPS-BDBPE detection, ${ }^{5}$ but the dopant agent and unpopularity of the APPI source made this method be not widely adopted for the further study of TBBPA and TBBPS derivatives. ${ }^{2}$ With respect to sample extraction and purification, accelerated solvent extraction (ASE), ${ }^{24}$ Soxhlet extraction, ${ }^{6}$ liquid-liquid extraction, ${ }^{25,26}$ solid phase extraction $(\mathrm{SPE})^{5}$ and gel permeation chromatography (GPC) ${ }^{10}$ have been used for the analysis of several specific TBBPA/S derivatives. However, the simultaneous determination of TBBPA, TBBPS, and their derivatives and byproducts is still a challenge because their polar diversity increases difficulties in extraction, purification and instrumental analysis. Moreover, there is no analytical method available for determination of some novel TBBPA analogs, such as TBBPA diol, TBBPA mono(2,3-dihydroxylpropyl ether) and TBBPA mono(2-bromoallyl ether) (TBBPA-MBAE). ${ }^{13}$

In this study, a comprehensive method for simultaneous determination of TBBPA/S and nine analogs in biological samples was developed using a single extract. Using the proposed method, the distribution patterns and tropic transfer properties of these compounds in various biological samples from the Chinese Bohai Sea were studied in detail. For the first time, TBBPA-MBAE and TBBPS-BDBPE were detected in biological samples and TBBPA-MBAE was identified as a byproduct. In addition, the trophic dilution tendencies of TBBPA/S analogs are being presented for the first time.

\section{MATERIALS AND METHODS}

Chemicals and Materials. The structures of TBBPA/S analogs are shown in Table 1. The byproducts, TBBPA-MAE (99\%), TBBPA-MBAE (98\%), TBBPA-MDBPE (98\%), TBBPS-MAE (99\%), TBBPS-MBAE (98\%) and TBBPSMDBPE (96\%), were synthesized in our laboratory and were further characterized by ${ }^{1} \mathrm{H}$ nuclear magnetic resonance $\left({ }^{1} \mathrm{HNMR}\right)$ and high resolution mass spectrometry (HRMS). ${ }^{14,15}$ The synthesis procedures, as well as the ${ }^{1} \mathrm{HNMR}$ and HRMS spectra of TBBPA-MBAE are shown in the Figure S1 and S2 (Supporting Information). TBBPA (50 $\mu \mathrm{g} / \mathrm{mL}$ in methanol), ${ }^{13} \mathrm{C}_{12}$-labeled TBBPA $(50 \mu \mathrm{g} / \mathrm{mL}$ in methanol) and $\mathrm{D}_{10}$-labeled TBBPA $(100 \mu \mathrm{g} / \mathrm{mL}$ in acetonitrile) were purchased from Cambridge Isotope Laboratories, Inc. (Massachusetts). TBBPA-BAE (99\%) and TBBPA-BDBPE (99\%) were purchased from Sigma-Aldrich. TBBPS (98\%) and TBBPS-BDBPE (99\%) were purchased from Beijing Apis Biotechnology Co., Ltd. All solid standards were dissolved in methanol at the concentration of $100 \mu \mathrm{g} / \mathrm{mL}$ and stored in a refrigerator at $4{ }^{\circ} \mathrm{C}$. Methanol, acetone, hexane and methylene dichloride (DCM) were all HPLC grade. Deionized water was generated by a Milli-Q advantage A10 system. Three solid commercial products of TBBPA-BAE and TBBPA-BDBPE were purchased from three BFR factories located in the south coastal area of the Chinese Bohai Sea to test the existence status of the monomodified byproducts.

Instrumental Parameters of HPLC-MS/MS and HPLC-DAD. HPLC-ESI-MS/MS (2695 HPLC, Quattro Ultima triple quadrupole mass spectrometer, Waters, Milford, MA) was used for determination of TBBPA, TBBPS, TBBPSBDBPE, TBBPA-MAE, TBBPA-MBAE, TBBPA-MDBPE, TBBPS-MAE, TBBPS-MBAE, and TBBPS-MDBPE under negative ionization mode, and the injection volume was 20 $\mu \mathrm{L}$. HPLC-DAD (Thermo Ultimate 3000, UV wavelength 214 $\mathrm{nm}$ ) was optimized for detection of TBBPA-BAE and TBBPA$\mathrm{BDBPE}$, and the injection volume was $30 \mu \mathrm{L}$. The detailed instrument parameters are described in Table 2 and Supporting Information.

\section{Table 2. Optimized ESI-MS/MS (Negative) Parameters}

\begin{tabular}{|c|c|c|c|c|c|}
\hline compounds & $\begin{array}{c}\text { cone } \\
(\mathrm{V})\end{array}$ & $\begin{array}{l}\text { quantitative } \\
\text { parent } \rightarrow \\
\text { daughter ions } \\
(\mathrm{m} / \mathrm{z})\end{array}$ & $\begin{array}{c}\text { coll } \\
\text { energy } \\
(\mathrm{eV})\end{array}$ & $\begin{array}{l}\text { qualitative } \\
\text { parent } \rightarrow \\
\text { daughter ions } \\
\underset{(m / z)}{(m / z)}\end{array}$ & $\begin{array}{c}\text { coll } \\
\text { energy } \\
(\mathrm{eV})\end{array}$ \\
\hline TВBPA & 45 & $542.7 \rightarrow 419.8$ & 45 & $540.7 \rightarrow 417.8$ & 45 \\
\hline TBBPA-D 10 & 45 & $552.6 \rightarrow 424.6$ & 45 & $550.6 \rightarrow 422.6$ & 45 \\
\hline TBBPA- ${ }^{13} \mathrm{C}_{12}$ & 45 & $554.6 \rightarrow 430.6$ & 45 & $556.6 \rightarrow 432.6$ & 45 \\
\hline TBBPS & 50 & $564.6 \rightarrow 249.8$ & 47 & $564.6 \rightarrow 78.9$ & 65 \\
\hline TBBPA-MAE & 35 & $582.7 \rightarrow 526.6$ & 43 & $580.7 \rightarrow 524.6$ & 43 \\
\hline TBBPA-MBAE & 40 & $660.7 \rightarrow 526.6$ & 45 & $662.7 \rightarrow 526.6$ & 45 \\
\hline $\begin{array}{l}\text { TBBPA- } \\
\text { MDBPE }\end{array}$ & 50 & $742.6 \rightarrow 526.6$ & 50 & $740.6 \rightarrow 524.6$ & 50 \\
\hline TBBPS-MAE & 35 & $604.7 \rightarrow 563.6$ & 38 & $602.7 \rightarrow 561.6$ & 38 \\
\hline TBBPS-MBAE & 35 & $682.6 \rightarrow 563.6$ & 30 & $684.6 \rightarrow 563.6$ & 30 \\
\hline $\begin{array}{l}\text { TBBPS- } \\
\text { MDBPE }\end{array}$ & 60 & $764.5 \rightarrow 563.6$ & 35 & $762.6 \rightarrow 561.6$ & 35 \\
\hline $\begin{array}{l}\text { TBBPS- } \\
\text { BDBPE }\end{array}$ & 60 & $764.5 \rightarrow 563.6$ & 35 & $762.6 \rightarrow 561.6$ & 35 \\
\hline
\end{tabular}

Sample Collection. The Chinese Bohai Sea, whose total area is $77.3 \times 10^{3} \mathrm{Km}^{2}$, is located in the northeast China and surrounded by 17 densely populated coastal cities. The coastal region surrounding the Chinese Bohai Sea has convenient transportation, intensive industry and a well-developed economy, and is famous for the Bohai Economic Rim. However, due to anthropogenic activities in the surrounding area, environmental quality has declined with increasing economic development over the past few decades. Relatively high concentrations of persistent organic pollutants and metals have been reported in different mollusks, phytoplankton/ seston, zooplankton, invertebrate, fish and seabird species of the Chinese Bohai Sea, such as polycyclic aromatic hydrocarbons (PAHs), polychlorinated biphenyls (PCB), polybromi- 
nated diphenylethers (PBDEs), organochlorine pesticides and mercury. ${ }^{14,27-32}$

In this study, a total of 97 biological samples (including 5 phycophyta species, 2 zooplankton species, 14 invertebrate species and 13 fish species) were collected from the coastal areas of the Chinese Bohai Sea near five cities, Dalian (DL), Huludao (HLD), Tianjin (TJ), Penglai (PL) and Yantai (YT), in July and November of 2012. Detailed information for each sample is presented in Table S1 (Supporting Information). The collected samples were transported to the laboratory on ice and cleaned with tap water and deionized water in the laboratory. The viscera and skin were excised and discarded. Approximately 100-500 g of wet soft tissue of each species collected at a single sampling site was homogenized in a blender to form a composite sample. The samples were freeze-dried, ground and stored at $-20{ }^{\circ} \mathrm{C}$ before analysis.

Sample Preparation. Each sample $(1.0 \mathrm{~g})$ was mixed with anhydrous sodium sulfate $(5 \mathrm{~g})$, spiked with ${ }^{13} \mathrm{C}_{12}$-labeled TBBPA (10 ng) surrogate standards, and extracted with 100 $\mathrm{mL} \mathrm{DCM}$ on an accelerated solvent extractor (Dionex ASE $350)$ at $100{ }^{\circ} \mathrm{C}$ and 1500 psi for 12 min with 3 cycles. The extraction solvent was then removed using rotary evaporator and solvent-exchanged to $0.5 \mathrm{~mL}$ of $\mathrm{DCM} /$ hexane $(1 / 1)$ and purified using GPC (DCM/hexane, 1/1; $38 \mathrm{~g} \mathrm{~S}$-X3 biobeads). The first $90 \mathrm{~mL}$ fraction of GPC obtained after loading the sample was discarded after which $100 \mathrm{~mL}$ of elution solvent was collected and concentrated to $0.5 \mathrm{~mL}$ with a rotary evaporator and a gentle stream of nitrogen. The sample was loaded onto a Supelclean LC-Si cartridge $(500 \mathrm{mg}, 6 \mathrm{~mL})$ and washed with 6 $\mathrm{mL} \mathrm{DCM} /$ hexane $(1 / 9, \mathrm{~V} / \mathrm{V}, \mathrm{F} 1)$, eluted with $10 \mathrm{~mL} \mathrm{DCM} /$ hexane $(1 / 3, \mathrm{~V} / \mathrm{V}, \mathrm{F} 2), 10 \mathrm{~mL} \mathrm{DCM} /$ hexane $(1 / 1, \mathrm{~V} / \mathrm{V}, \mathrm{F} 3)$ and $10 \mathrm{~mL} \mathrm{NH} \mathrm{NH}_{3} \cdot \mathrm{H}_{2} \mathrm{O}$ /acetone $(0.5 \%, \mathrm{~V} / \mathrm{V}, \mathrm{F} 4)$. TBBPA-BAE and TBBPA-BDBPE existed in fraction F2; TBBPS-BDBPE and a trace amount of TBBPA, TBBPA-MAE, TBBPA-MBAE, and TBBPA-MDBPE existed in fraction F3; most of TBBPA, TBBPS and the monomodified byproducts existed in fraction F4. F2, F3, and F4 were each concentrated with a gentle stream of nitrogen. F2 was solvent-changed to $50 \mu \mathrm{L}$ methanol and analyzed with HPLC-DAD. F4 was solvent-changed to $1 \mathrm{~mL}$ $\mathrm{DCM} /$ hexane $(1 / 1, \mathrm{~V} / \mathrm{V})$, further purified with ENVI-Carb cartridge $(500 \mathrm{mg}, 6 \mathrm{~mL})$ and eluted with $10 \mathrm{~mL} \mathrm{NH} \cdot \mathrm{H}_{2} \mathrm{O} /$ acetone $(0.5 \%, \mathrm{~V} / \mathrm{V})$ which combined with $\mathrm{F} 3$ from the LC-Si cartridge. After concentration with a stream of nitrogen, this fraction was solvent changed to $200 \mu \mathrm{L}$ methanol, spiked with internal standard $\mathrm{D}_{10}$-labeled TBBPA $(10 \mathrm{ng})$ and analyzed with HPLC-ESI-MS/MS.

Trophic Level (TL) and Trophic Magnification Factor (TMF) Determination. The TL and TMF were calculated based on the stable isotope analysis for $\delta^{15} \mathrm{~N}$ which was determined with a Thermo DELTA V Advantage isotope ratio mass spectrometer interfaced to a Flash EA1112 HT elemental analyzer (Thermo Fisher). The specific details are described in Supporting Information.

\section{RESULTS AND DISCUSSION}

Optimization of Instrumental Parameters. For ESIMS/MS parameters' optimization, individual standard solution for each target compound was injected to obtain mass spectra under the negative ionization mode. For TBBPA, TBBPAMAE, TBBPA-MBAE, TBBPA-MDBPE, TBBPS, TBBPSMAE, TBBPS-MBAE, and TBBPS-MDBPE generated [M$\mathrm{H}]^{-}$ions as the parent ions. For TBBPS-BDBPE, the parent ion $764.5\left(\left[\mathrm{M}-\mathrm{C}_{3} \mathrm{H}_{5} \mathrm{Br}_{2}\right]^{-}\right)$was generated from ether bond fragmentation. The optimized quantitative and qualitative ions are shown in Table 2.

Using HPLC-ESI-MS/MS, TBBPA, TBBPA-MAE, TBBPA-MBAE, TBBPA-MDBPE, TBBPS, TBBPS-MAE, TBBPS-MBAE, TBBPS-MDBPE, and TBBPS-BDBPE could be determined simultaneously (Figure 1). Herein, we tried to

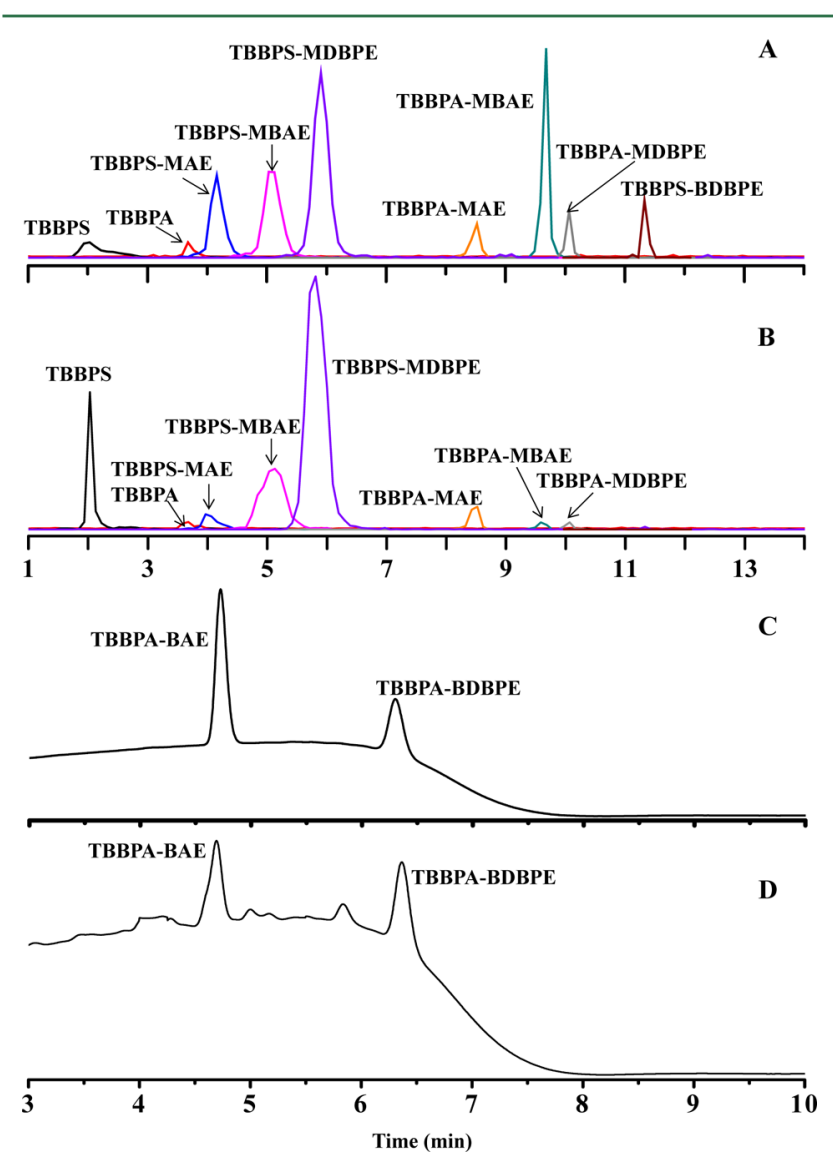

Figure 1. HPLC-ESI ${ }^{-}$-MS/MS (A, B) and HPLC-UV (C, D, 214 nm) chromatograms of TBBPA/S and nine analogs. A: standard solution (2 $\mathrm{ng} / \mathrm{mL}$ ). B: Grateloupia ramosissima sample collected from Huludao. C: standard solution $(1 \mu \mathrm{g} / \mathrm{mL})$. D: Portunus trituberculatus sample collected from Dalian.

analyze TBBPA-BAE and TBBPA-BDBPE by APPI-MS of Agilent Technologies (6460 Triple Quadrupole MS/MS) and Waters (Quattro Ultima Triple Quadrupole MS/MS), but the MS signals were very low. After optimization of pretreatment method, HPLC coupled with a UV detector was previously used for the analysis of TBBPA-BAE and TBBPA-BDBPE. ${ }^{33}$ In this study, by using HPLC-DAD, TBBPA-BAE and TBBPABDBPE could be separated completely with other interference peaks specifically and they were quantified by the peak area (UV $214 \mathrm{~nm}$, Figure 1).

Optimization of the SPE Method for TBBPA/S Analogs by Single Extract. Three kinds of SPE cartridges (Supelclean ENVI-Carb, LC-Florisil ${ }^{\circledR}$, LC-Si, $500 \mathrm{mg}, 6 \mathrm{~mL}$ ) were used to evaluate the cleaning and concentration efficiencies after GPC purification. A variety of eluents, including hexane, different proportions of hexane/DCM, DCM, acetone, $0.5 \% \mathrm{NH}_{3} \cdot \mathrm{H}_{2} \mathrm{O}$ / acetone were evaluated for the elution of the target compounds from these three cartridges. TBBPA-BAE and TBBPA-BDBPE showed the lowest absorption capacity with the LC-Si cartridges, followed by TBBPS-BDBPE, TBBPA-MDBPE, 
TBBPA-MBAE, and TBBPA-MAE. TBBPA, TBBPS, TBBPSMAE, TBBPS-MBAE and TBBPS-MDBPE showed the greatest absorption capacity with LC-Si cartridges and could only be eluted using acetone. We minimized the matrix interference that could affect HPLC-DAD signals by using a weak polar eluent, DCM/hexane $(1 / 3, \mathrm{~V} / \mathrm{V})$, for the elution of TBBPABAE and TBBPA-BDBPE. It was found that $10 \mathrm{~mL} 0.5 \% \mathrm{NH}_{3}$. $\mathrm{H}_{2} \mathrm{O}$ /acetone could elute the other loaded compounds efficiently, but interferences were also eluted from LC-Si cartridges. Therefore, ENVI-Carb cartridge was subsequently applied for the concentration and purification of high polar TBBPA, TBBPS and their byproducts. Meanwhile, we found that $20 \mathrm{~mL}$ acetone was better than $10 \mathrm{~mL}$ as an eluent for the recovery of TBBPA, TBBPS and byproducts. Because phenols are weak acids, a small amount of a weak basic can be helpful for the elution of the target compounds. Thus, $10 \mathrm{~mL} 0.5 \%$ $\mathrm{NH}_{3} \cdot \mathrm{H}_{2} \mathrm{O}$ /acetone was sufficient for completely eluting TBBPA, TBBPS and their byproducts. The residue obtained from F3 using LC-Si cartridge could be further purified with an ENVI-Carb cartridge to eliminate most pigment and interference. The elution of TBBPS-BDBPE from LC-Si cartridges was better than from ENVI-Carb cartridges. The combination of LC-Si and ENVI-Carb cartridges provided an efficient pretreatment method for all of the 11 compounds. Finally, the purification and concentration for these 11 diversely polar compounds were effectively completed by single ASE extraction.

Method Performance. TBBPA, TBBPS, TBBPS-BDBPE and their byproducts in samples were identified by retention time, quantitative ions and qualitative ions comparison with the corresponding standards. Quantification of TBBPA, TBBPAMAE, TBBPA-MBAE and TBBPA-MDBPE, TBBPS, TBBPSMAE, TBBPS-MBAE, TBBPS-MDBPE, and TBBPS-BDBPE in the environmental samples was performed using the internal standard method and corrected using surrogate recovery. The standard curve was established between the peak area ratios of the compounds/internal standard and the concentrations, and was used to quantify the target compounds in collected samples. Procedural blanks, standard solution (10 ppb), replicate sample and methanol were tested with each batch of samples to check for potential interference and crosscontamination. The selection of the quantitative and qualitative ions of ${ }^{13} \mathrm{C}_{12}$-labeled TBBPA and $\mathrm{D}_{10}$-labeled TBBPA could avoid the interference between these two substances in MS analysis (detailed discussion in Supporting Information). The instrument detection limits (IDLs) and instrument quantification limits (IQLs) were determined by $3 / 1$ and $10 / 1$ signal-tonoise value $(\mathrm{S} / \mathrm{N})$ separately. The method quantification limits (MQLs) and method detection limits (MDLs) were based on replicate analyses $(n=8)$ of blank river fish sample spiked at concentrations of $1-5$ times of the IQLs and the IDLs, respectively. MQLs were defined as the minimum amount of analyte producing a peak with $S / N=10 / 1$ and MDLs were defined as $\mathrm{S} / \mathrm{N}=3 / 1$. MDLs, MQLs, recoveries and matrix effects are all shown in Table S2. The IDLs of TBBPA, TBBPAMAE, TBBPA-MBAE, TBBPA-MDBPE, TBBPS, TBBPSMAE, TBBPS-MBAE, TBBPS-MDBPE, and TBBPS-BDBPE ranged from 0.02 to $2 \mathrm{pg}$, and the IQLs ranged from 0.07 to 7 pg (Table S2). The MDLs of TBBPA, TBBPA-MAE, TBBPAMBAE, TBBPA-MDBPE, TBBPS, TBBPS-MAE, TBBPSMBAE, TBBPS-MDBPE, and TBBPS-BDBPE ranged from $0.6 \mathrm{pg} / \mathrm{g} \mathrm{dw}$ to $100 \mathrm{pg} / \mathrm{g} \mathrm{dw}$, and the MQLs ranged from 2.0 $\mathrm{pg} / \mathrm{g} \mathrm{dw}$ to $300 \mathrm{pg} / \mathrm{g}$ dw (Table S2).
TBBPA-BAE and TBBPA-BDBPE were quantified with an external method, and the results were not corrected using surrogate recovery. The instrument detection limits (IDLs) of TBBPA-BAE and TBBPA-BDBPE were 300 and 3000 pg, and the instrument quantification limits (IQLs) were 900 and 9000 pg. The MDL and MQL for TBBPA-BAE were $700 \mathrm{pg} / \mathrm{g} \mathrm{dw}$ and $2300 \mathrm{pg} / \mathrm{g} \mathrm{dw}$ which were much better than those of APCIMS/MS (MDL $20000 \mathrm{pg} / \mathrm{g} \mathrm{dw}$ and MQL $80000 \mathrm{pg} / \mathrm{g} \mathrm{dw}$ ) applied for the analysis of biological samples. ${ }^{14}$ Meanwhile, the MDL and MQL for TBBPA-BDBPE in biological samples, which benefiting from the optimized pretreatment method, were $6000 \mathrm{pg} / \mathrm{g} \mathrm{dw}$ and $20000 \mathrm{pg} / \mathrm{g} \mathrm{dw}$, much lower than the previously reported values of $50000 \mathrm{pg} / \mathrm{g} \mathrm{dw}$ and $180000 \mathrm{pg} / \mathrm{g}$ dw. ${ }^{14}$ Thus, it appears optimized pretreatment method can compensate for insufficient sensitivity of the detector.

The recoveries ranged from $75 \%$ to $106 \%$ for Paralichthys olivaceus (Par) samples $(n=4)$ at different spiking amounts $(1$, 10,100 , and $1000 \mathrm{ng} / \mathrm{g} \mathrm{dw}$ ). The method was further evaluated with five different matrices including Mugil soiuy (MugS), Crassostrea talienwhanensis (Ost), Portunus trituberculatus (Por), Metapenaeus ensis (Met) and Ulva pertusa (Ulv) at a spiking amount of $10 \mathrm{ng} / \mathrm{g} \mathrm{dw}$ (for TBBPA-BAE and TBBPA-BDBPE were $100 \mathrm{ng} / \mathrm{g} \mathrm{dw})$. The recoveries ranged from $65 \%$ to $108 \%$ and the standard deviations were all less than $10 \%$. The matrix effects ranged from 0.88 to 1.15 at different spiking concentrations $(1,10,100$, and $1000 \mathrm{ng} / \mathrm{mL})$, which indicated that signal enhancement or signal suppression for the target compounds could be ignored. These results indicated that we had developed an effective method for the simultaneous analysis of TBBPA, TBBPS, and their derivatives and byproducts in various biological samples. The single extract pretreatment procedure can reduce the amount of sample required for analysis and improve analysis efficiency. This method could also be widely applied for further study of the transportation and transformation of TBBPA/S analogs in the environment.

TBBPA/S Analogs in Commercial Products. Since they are produced with TBBPA/S as raw materials, the commercial products of BFRs contain hypothetical monomodified compounds as impurities when the reactions of TBBPA/S with corresponding reagents are incomplete. ${ }^{13}$ In our previous studies, TBBPA-MAE and TBBPA-MDBPE were identified as the byproducts of commercial TBBPA-BAE and TBBPABDBPE; ${ }^{14}$ TBBPS-MAE, TBBPS-MBAE, and TBBPS-MDBPE were also identified as byproducts of commercial TBBPSBDBPE. $^{15}$ In this study, TBBPA-MAE and TBBPA-MDBPE were also detectable in the three commercial TBBPA-BAE and TBBPA-BDBPE products with average concentrations of $9.2 \mathrm{~g} /$ $\mathrm{kg}$ and $5.4 \mathrm{~g} / \mathrm{kg}$, respectively. For the first time, TBBPA-MBAE was detectable in the three commercial TBBPA-BAE and TBBPA-BDBPE, with average concentrations of $10 \mathrm{mg} / \mathrm{kg}$ and $5 \mathrm{mg} / \mathrm{kg}$, respectively, concentrations that were much lower than those of TBBPA-MAE and TBBPA-MDBPE in commercial products. The results indicated TBBPA-MBAE was also a byproduct of TBBPA derivatives.

TBBPA/S Analogs in Biological Samples Collected from the Chinese Bohai Sea. Using the proposed method, 11 target compounds in the collected marine biological samples were determined and reported on a lipid weight (lw) basis (Table S1 and Table 3). The concentrations of individual compound, TBBPA and its analogs, ranged from ND to 2782.8 $\mathrm{ng} / \mathrm{g} \mathrm{lw}$; for TBBPS and its analogs concentrations ranged from ND to $927.8 \mathrm{ng} / \mathrm{g}$ lw. Relative high concentrations of TBBPS 
Table 3. Descriptive Statistics of the Target TBBPA/S and Analogs in the Collected Marine Biological Samples

\begin{tabular}{|c|c|c|c|c|c|}
\hline & $\begin{array}{l}\text { con. range } \\
(\mathrm{ng} / \mathrm{g} \mathrm{lw})\end{array}$ & $\begin{array}{c}\text { media } \\
(\mathrm{ng} / \mathrm{g} \\
\mathrm{lw})\end{array}$ & $\begin{array}{c}\text { geometric } \\
\text { mean (ng/g } \\
\text { lw) }\end{array}$ & $\begin{array}{c}\text { mean } \\
(\mathrm{ng} / \mathrm{g} \\
\mathrm{lw})\end{array}$ & $\begin{array}{l}\text { detection } \\
\text { frequency }\end{array}$ \\
\hline ТВBPA & ND-207.3 & 7.3 & 6.9 & 24.2 & $87 \%$ \\
\hline $\begin{array}{l}\text { TBBPA- } \\
\text { MAE }\end{array}$ & ND-252.1 & 4.6 & 4.8 & 4.8 & $98 \%$ \\
\hline $\begin{array}{l}\text { TBBPA- } \\
\text { MBAE }\end{array}$ & ND-19.7 & 0.1 & 0.1 & 0.1 & $58 \%$ \\
\hline $\begin{array}{l}\text { TBBPA- } \\
\text { MDBPE }\end{array}$ & ND-49.2 & 3.1 & 2.9 & 2.9 & $95 \%$ \\
\hline TBBPS & ND-927.8 & 21.8 & 15.8 & 15.8 & $97 \%$ \\
\hline $\begin{array}{l}\text { TBBPS- } \\
\text { MAE }\end{array}$ & ND-108.3 & 2.9 & 2.1 & 2.1 & $87 \%$ \\
\hline $\begin{array}{l}\text { TBBPS- } \\
\text { MBAE }\end{array}$ & $0.1-151.1$ & 5.6 & 5.5 & 5.5 & $100 \%$ \\
\hline $\begin{array}{l}\text { TBBPS- } \\
\text { MDBPE }\end{array}$ & ND-68.2 & 2.5 & 2.7 & 2.7 & $97 \%$ \\
\hline $\begin{array}{l}\text { TBBPA- } \\
\text { BAE }\end{array}$ & ND-898.4 & NQ & 8.9 & 8.9 & $26 \%$ \\
\hline $\begin{array}{l}\text { TBBPA- } \\
\text { BDBPE }\end{array}$ & ND-2782.8 & NQ & 39.1 & 39.1 & $4 \%$ \\
\hline $\begin{array}{l}\text { TBBPS- } \\
\text { BDBPE }\end{array}$ & ND-55.5 & NQ & 0.3 & 0.3 & $31 \%$ \\
\hline
\end{tabular}

NQ: not quantified. For the statistical analysis, ND was treated as half value of MDL and NQ was treated as half value of MQL.

were observed, with a concentration range of ND to $927.8 \mathrm{ng} / \mathrm{g}$ lw. The concentration ranges of TBBPA-MAE, TBBPA-MBAE, TBBPA-MDBPE, TBBPS-MAE, TBBPS-MBAE, and TBBPSMDBPE were ND to $252.1 \mathrm{ng} / \mathrm{g} \mathrm{lw}$, ND to $19.7 \mathrm{ng} / \mathrm{g}$ lw, ND to $49.2 \mathrm{ng} / \mathrm{g} \mathrm{lw}$, ND to $108.3 \mathrm{ng} / \mathrm{g} \mathrm{lw}, 0.1$ to $151.1 \mathrm{ng} / \mathrm{g}$ lw and $\mathrm{ND}$ to $68.2 \mathrm{ng} / \mathrm{g}$ lw, respectively. Except for TBBPA-MBAE, whose detection frequency is $58 \%$, the detection frequencies for TBBPA, TBBPS and the monomodified byproducts were all higher than $86 \%$. For TBBPA-BAE, TBBPA-BDBPE, and TBBPS-BDBPE, the concentrations ranged from ND to 898.4 $\mathrm{ng} / \mathrm{g} \mathrm{lw}, \mathrm{ND}$ to $2782.8 \mathrm{ng} / \mathrm{g} \mathrm{lw}$ and ND to $55.5 \mathrm{ng} / \mathrm{g} \mathrm{lw}$, respectively. The detection frequencies for TBBPA-BAE, TBBPA-BDBPE, and TBBPS-BDBPE were $26 \%, 4 \%$, and $31 \%$, respectively. The lower detection frequencies for these three derivatives, which are directly produced by factories, may be due to two factors: first, the rapid degradation or elimination rates of the derivatives, which have been reported for TBBPABDBPE when tested with zebrafish, ${ }^{34}$ and second, the low detection sensitivity of TBBPA-BAE and TBBPA-BDBPE. The concentration levels and detection frequencies of TBBPAMAE, TBBPA-MDBPE, TBBPS-MAE, TBBPS-MBAE, and TBBPS-MDBPE were higher in this study than those in mollusk samples collected from coastal areas of the Chinese Bohai Sea. ${ }^{14,15}$ The detection frequencies of TBBPA, TBBPS and the monomodified byproducts were comparable to other BFRs that were detected in the same area, such as PBDEs and hexabromocyclododecane (HBCD). ${ }^{31}$ With the wide use of TBBPA/S, these byproducts have become the prevailing contaminants in this regions and their concentrations are gradually increasing.

Trophic Transfer of TBBPA, TBBPS, and Byproducts. Regression and correlation analysis between the lipid-corrected concentrations and the TLs were conducted for TBBPA, TBBPS, TBBPA-MAE, TBBPA-MDBPE, TBBPS-MAE, TBBPS-MBAE, and TBBPS-MDBPE (detection frequency $>86 \%$ ) using 86 samples with more than two replicate samples. In case of the values lower than MDLs and between MDLs and
MQLs, the concentrations were treated as half of MDLs and half of MQLs, respectively. In Figure 2, negative correlations

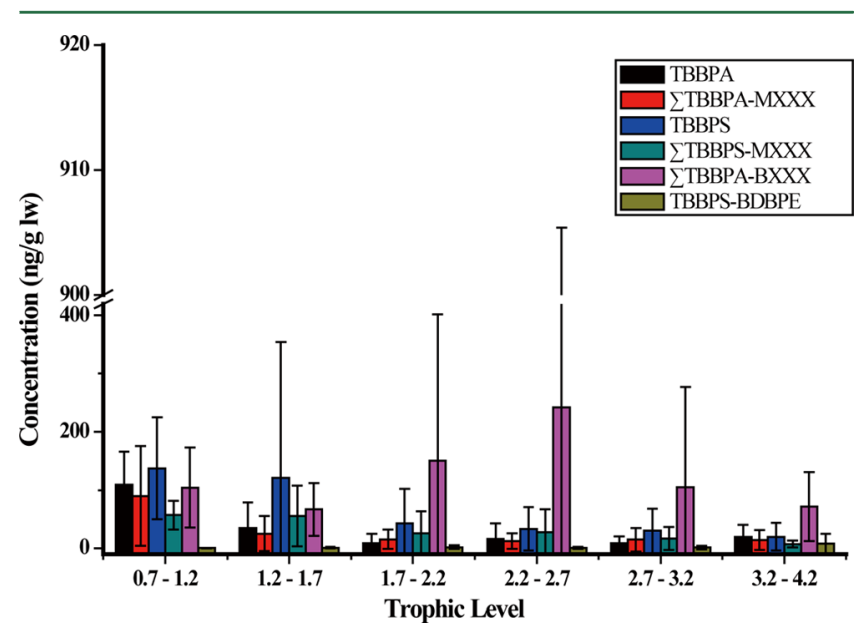

Figure 2. Concentration (ng/g lw) distribution for TBBPA/S analogs with trophic levels. $\sum$ TBBPA-MXXX is the total concentration of TBBPA-MAE, TBBPA-MBAE and TBBPA-MDBPE; $\sum$ TBBPSMXXX is the total concentration of TBBPS-MAE, TBBPS-MBAE, and TBBPS-MDBPE; $\sum$ TBBPA-BXXX is the total concentration of TBBPA-BAE and TBBPA-BDBPE.

were observed between the concentrations of TBBPA, the total concentration of TBBPA byproducts, TBBPS, the total concentration of TBBPS byproducts and the TLs. The total concentration of TBBPA-BAE and TBBPA-BDBPE increased with the trophic level between TL 0.7 and 2.7, but decreased between TL 2.7 and 3.2. The concentration of TBBPS-BDBPE remained stable and no significant correlation between TL was observed. The levels of target compounds detected in mollusk and plant samples were higher than those found in fish samples. As shown in Figure 3, logarithmic lipid-normalized concentrations of all target compounds decreased significantly with increasing TLs and the correlations between these two factors were significant $(p<0.05$, Table S3.). The TMF values for the target compounds ranged from 0.31 to 0.55 , as shown in Figure 3. These results indicated that TBBPA, TBBPS, and their byproducts undergo trophic dilution in the Chinese Bohai Sea food web, which is similar to a number of other organic pollutants including tris(2,3-dibromopropyl) isocyanurate, HBCD, PAHs, dibenzo-p-dioxin (PCDD), hexachlorocyclohexane and PBDEs. ${ }^{28-31}$

The biomagnification potential of pollutants is often evaluated using the octanol-water partition coefficient (log $\left.K_{\text {ow }}\right)$, which is estimated with the Estimation Programs Interface (EPI) Suite software. ${ }^{8,13,30,31}$ Generally, a compound with $\log K_{\text {ow }}>5$ is considered to be potentially bioaccumulative. $8,13,35,36$ Although the $\log K_{\text {ow }}$ values of the monomodified byproducts were lower than the corresponding derivatives, they all showed $\log K_{\mathrm{ow}}>5$, indicating their potential bioaccumulative abilities. ${ }^{8}$ For TBBPA, TBBPS and the byproducts, the $\log K_{\text {ow }}$ ranged from 5.21 to $9.36^{15}$ and no significant relationship was observed between $\log K_{\text {ow }}$ values and TMFs. However, some other POPs with comparable log $K_{\text {ow }}$ values, such as DDT, hexachlorobenzene and non- and mono-ortho PCBs, exhibited positive correlation with TLs in the Chinese Bohai Sea marine food web: the TMF values of these substances were all higher than $1 .^{27,28}$ These results indicate that the $\log K_{\mathrm{ow}}$ is not the only factor influencing 

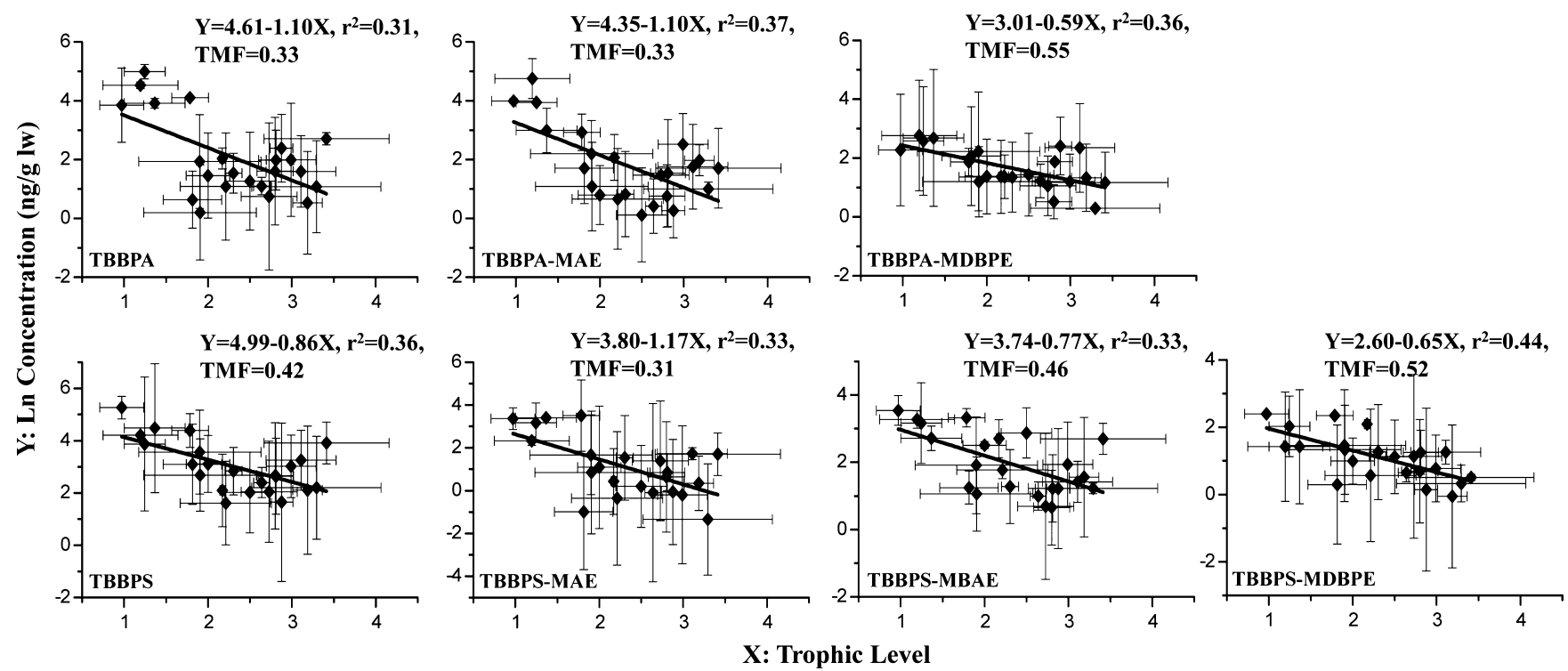

Figure 3. Logarithmic concentrations ( $\mathrm{ng} / \mathrm{g} \mathrm{lw}$ ) of target compounds and trophic level relationships for marine biological samples from the Chinese Bohai Sea. Linear regression lines were used for TMF calculation.

trophic transfer behavior of TBBPA, TBBPS and their byproducts, and that there are other determining factors at work, such as assimilation efficiencies, elimination rate, degradation rate and metabolic transformation.

Environmental Significance. The correlation analyses between TBBPA, TBBPS and the byproducts revealed that these similarly structured compounds have similar sources and similar environmental fates. The Spearman correlation analyses for TBBPA, TBBPA-MAE, TBBPA-MDBPE, TBBPS, TBBPSMAE, TBBPS-MBAE, and TBBPS-MDBPE were conducted and the $p$ values of Spearman's rank correlation test were shown in Table S3. Concentrations of TBBPA were significantly correlated with that of TBBPA-MAE $(p<0.01)$, and correlated with that of TBBPA-MDBPE $(p<0.05)$. Concentrations of TBBPA-MAE were significantly correlated with that of TBBPA-MDBPE $(p<0.01)$. The concentration correlations between each of the other TBBPS and its three byproducts, TBBPS-MAE, TBBPS-MBAE, and TBBPSMDBPE were all significant $(p<0.01)$. The results implied that TBBPA and TBBPA byproducts, and TBBPS and TBBPS byproducts, have the similar uptake pathways and kinetics. Significant correlations $(p<0.01)$ were also observed between TBBPA and TBBPS, as well as between TBBPA byproducts and TBBPS byproducts except for TBBPA-MDBPE and TBBPS-MAE. These relationships indicated TBBPA and TBBPS analogs were likely accumulated from similar sources, which are assumed to be located around the south costal area of the Chinese Bohai Sea. ${ }^{14}$

The incomplete reaction for the commercial products resulted in the occurrence of byproducts. For the TBBPA/S derivatives, the ether bond linked structures account for most products. The hydroxylated byproducts coproduced with commercial products seemed to be an important source of novel contaminants, not only for TBBPA and TBBPS, but also for other ether bond linked BFRs, such as 2,4,6-tris $(2,4,6$ tribromophenoxy)-1,3,5-triazine. ${ }^{37}$ Many byproducts originating from widely used BFRs have been found to exist in the environment and have been shown to have more severe environmental risks than the derivatives. ${ }^{15,38}$
The bioaccumulative capacity and transformation possibility could influence the distribution of the byproducts and derivatives in biological samples. The proportions for TBBPA-MAE and TBBPA-MDBPE in commercial TBBPABAE and TBBPA-BDBPE were $9.2 \times 10^{-03}: 1$ and $5.4 \times$ $10^{-03}: 1$, respectively. The proportions for TBBPA-MBAE in commercial TBBPA-BAE and TBBPA-BDBPE were $1.0 \times$ $10^{-05}: 1$ and $5.0 \times 10^{-06}: 1$, respectively. The proportions for TBBPS-MAE, TBBPS-MBAE and TBBPS-MDBPE in commercial TBBPS-BDBPE were $2.8 \times 10^{-05}: 1,8.7 \times 10^{-05}: 1$ and $3.9 \times 10^{-04}: 1$, respectively. ${ }^{15}$ In the marine biota samples TBBPA-BAE was detectable in 25 samples. Only one sample showed the ratio $\left(6.2 \times 10^{-03}: 1\right)$ for TBBPA-MAE:TBBPABAE lower than $9.2 \times 10^{-03}: 1$. Eleven samples showed ratios ranging from $1.4 \times 10^{-02}: 1$ to $8.2 \times 10^{-02}: 1$ and 13 samples showed ratios ranging from $1.0 \times 10^{-01}: 1$ to $8.6 \times 10^{-01}: 1$. TBBPA-BDBPE was detectable in four samples of which the ratios for TBBPA-MDBPE:TBBPA-BDBPE were $1.9 \times 10^{-03}: 1$, $1.5 \times 10^{-02}: 1,5.0 \times 10^{-02}: 1$ and $5.1 \times 10^{-02}: 1$, respectively. The proportions for TBBPA-MBAE:TBBPA-BAE and TBBPAMBAE:TBBPA-BDBPE in biological samples ranged from 5.2 $\times 10^{-04}: 1$ to $4.3 \times 10^{-02}: 1$ and from $1.9 \times 10^{-04}: 1$ to $4.4 \times$ $10^{-03}: 1$. The ratios for TBBPS-MAE, TBBPS-MBAE, and TBBPS-MDBPE to TBBPS-BDBPE in biological samples ranged from $7.9 \times 10^{-02}: 1$ to $39.0: 1,4.5 \times 10^{-02}: 1$ to $63.3: 1$ and $7.9 \times 10^{-02}: 1$ to $25.0: 1$, respectively. Most samples have a byproduct-to-derivative proportion that is higher than the proportion observed that in the commercial product. The first reason appears to be the higher bioaccumulation/bioconcentration capacities of the byproducts and the higher elimination rates of the derivatives. The bioconcentration factor (BCF) values of the byproducts were higher than the derivatives (Table 1), indicating the higher bioconcentration potential of the byproducts. The second reason for the ratio variation may be that the derivatives transform to the monomodified byproducts in the bodies of marine organism if the BFRs factories located near the Chinese Bohai Sea costal are the pollution sources. TBBPA-BAE, TBBPA-BDBPE, and TBBPS$\mathrm{BDBPE}$ are all formed by the ether bond, whose cleavage has been identified as an important degradation pathway for the 
emergence of hydroxylated BFRs in the environment. ${ }^{16}$ Ether bond-linked compounds are sensitive to bacterial degradation, thermal degradation and UV irradiation, and cleavage of the ether bond in these compounds forms phenols, including bromophenols. ${ }^{16-18,39-42}$ Currently, TBBPA-MAE and TBBPA-MDBPE have been identified as degradation products of TBBPA-BAE and TBBPA-BDBPE according to the results of UM-PPS (http://umbbd.msi.umn.edu/predict/).,14 TBBPAMAE, TBBPA-MDBPE, and TBBPS-MDBPE can be generated as the degradation products through the cleavage of a single ether bond in TBBPA-BAE, TBBPA-BDBPE, and TBBPSBDBPE, respectively. ${ }^{13-15}$ TBBPA and TBBPS are common degradation products that occur with the cleavage of two ether bonds in TBBPA and TBBPS derivatives. Although the environmental transformation of TBBPA has been well studied, the degradation pathways and mechanism of TBBPA/S derivatives in the environment are still unknown which deserve further investigation.

In this study, TBBPA/S and nine analogs were positively detected in various biological samples collected from the Chinese Bohai Sea. TBBPA/S and five analogs, including TBBPA-MAE, TBBPA-MDBPE, TBBPS-MAE, TBBPS-MBAE, and TBBPS-MDBPE, showed trophic dilution tendencies. These five analogs, together with the identified TBBPA$\mathrm{MBAE}$, can be generated not only as byproducts but also as probable transformation products of commercial TBBPA/S products. This is the first time the trophic transfer properties of novel TBBPA/S byproducts have been reported in the marine environment. However, examination of the fate and risks of TBBPA-BAE, TBBPA-BDBPE, and TBBPS-BDBPE in the environment is still in the start stage. More research concerning the exposure, uptake and transformation pathways and degradation products is still needed. As in this view, the proposed method has great potential for application in future studies of the environmental behavior and risks of emerging TBBPA/S derivatives.

\section{ASSOCIATED CONTENT}

\section{S Supporting Information}

The Supporting Information is available free of charge on the ACS Publications website at DOI: 10.1021/acs.est.5b06378.

Synthesis routines, methods, ${ }^{1} \mathrm{HNMR}$ data and spectrum, HRMS data and spectrum for TBBPA-MBAE; TL and TMF calculation method; detailed sample information; concentration (lipid corrected) of all samples; instrument parameters; method performance data; the correlation test results; selection of quantitative and qualitative ions of ${ }^{13} \mathrm{C}_{12}$-labeled TBBPA and $\mathrm{D}_{10}$-labeled TBBPA (PDF)

\section{AUTHOR INFORMATION}

\section{Corresponding Author}

*Phone/fax: +86-10-62849129; e-mail: jbshi@rcees.ac.cn.

\section{Notes}

The authors declare no competing financial interest.

\section{ACKNOWLEDGMENTS}

We thank Mei Meng, Dawei Lu and Chengbin Liu for their assistance in collecting samples. This work was supported by the National Basic Research Program of China (No. 2015CB453100), the National Natural Science Foundation of China (Nos. 21377155 and 21321004), and the Strategic
Priority Research Program of the Chinese Academy of Sciences (No. XDB14010400).

\section{REFERENCES}

(1) Covaci, A.; Harrad, S.; Abdallah, M. A. E.; Ali, N.; Law, R. J.; Herzke, D.; de Wit, C. A. Novel brominated flame retardants: A review of their analysis, environmental fate and behaviour. Environ. Int. 2011, 37 (2), 532-556.

(2) Covaci, A.; Voorspoels, S.; Abdallah, M. A. E.; Geens, T.; Harrad, S.; Law, R. J. Analytical and environmental aspects of the flame retardant tetrabromobisphenol-A and its derivatives. J. Chromatogr. A 2009, 1216 (3), 346-363.

(3) European Union Risk Assessment Report. EU RAR CAS No. 7994-7 EINECS: 201-236-9 2,2',6,6'-tetrabromo-4,4'-isopropylidenediphenol (tetrabromobisphenol A or TBBPA) Part II Human Health; European Commission Joint Research Centre: EUR 22161 EN 4th Priority List. 2006; Vol. 63, http://europa.eu.int.

(4) Ali, N.; Harrad, S.; Goosey, E.; Neels, H.; Covaci, A. "Novel" brominated flame retardants in Belgian and UK indoor dust: Implications for human exposure. Chemosphere 2011, 83 (10), 1360-1365.

(5) Letcher, R. J.; Chu, S. High-sensitivity method for determination of tetrabromobisphenol-S and tetrabromobisphenol-A derivative flame retardants in Great Lakes herring gull eggs by liquid chromatographyatmospheric pressure photoionization-tandem mass spectrometry. Environ. Sci. Technol. 2010, 44 (22), 8615-8621.

(6) Shi, T.; Chen, S. J.; Luo, X. J.; Zhang, X. L.; Tang, C. M.; Luo, Y.; Ma, Y. J.; Wu, J. P.; Peng, X. Z.; Mai, B. X. Occurrence of brominated flame retardants other than polybrominated diphenyl ethers in environmental and biota samples from southern China. Chemosphere 2009, 74 (7), 910-916.

(7) Nyholm, J. R.; Grabic, R.; Arp, H. P. H.; Moskeland, T.; Andersson, P. L. Environmental occurrence of emerging and legacy brominated flame retardants near suspected sources in Norway. Sci. Total Environ. 2013, 443, 307-314.

(8) Howard, P. H.; Muir, D. C. G. Identifying new persistent and bioaccumulative organics among chemicals in commerce. Environ. Sci. Technol. 2010, 44 (7), 2277-2285.

(9) Qu, G.; Shi, J.; Wang, T.; Fu, J.; Li, Z.; Wang, P.; Ruan, T.; Jiang, G. Identification of tetrabromobisphenol A diallyl ether as an emerging neurotoxicant in environmental samples by bioassay-directed fractionation and HPLC-APCI-MS/MS. Environ. Sci. Technol. 2011, 45 (11), 5009-5016.

(10) Nyholm, J. R.; Norman, A.; Norrgren, L.; Haglund, P.; Andersson, P. L. Maternal transfer of brominated flame retardants in zebrafish (Danio rerio). Chemosphere 2008, 73 (2), 203-208.

(11) Knudsen, G. A.; Jacobs, L. M.; Kuester, R. K.; Sipes, I. G. Absorption, distribution, metabolism and excretion of intravenously and orally administered tetrabromobisphenol A 2,3-dibromopropyl ether in male Fischer-344 rats. Toxicology 2007, 237 (1-3), 158-167.

(12) Eljarrat, E.; Feo, M. L.; Barcelo, D. Degradation of brominated flame retardants. In Brominated Flame Retardants; Eljarrat, E., Barcelo, D., Eds.; Springer-Verlag: Berlin Heidelberg, 2011; pp 188-202.

(13) Howard, P. H.; Muir, D. C. G. Identifying new persistent and bioaccumulative organics among chemicals in commerce. III: Byproducts, impurities, and transformation products. Environ. Sci. Technol. 2013, 47 (10), 5259-5266.

(14) Qu, G.; Liu, A.; Wang, T.; Zhang, C.; Fu, J.; Yu, M.; Sun, J.; Zhu, N.; Li, Z.; Wei, G.; Du, Y.; Shi, J.; Liu, S.; Jiang, G. Identification of tetrabromobisphenol A allyl ether and tetrabromobisphenol A 2,3dibromopropyl ether in the ambient environment near a manufacturing site and in mollusks at a coastal region. Environ. Sci. Technol. 2013, 47 (9), 4760-4767.

(15) Liu, A.; Tian, Y.; Yin, N.; Yu, M.; Qu, G.; Shi, J.; Du, Y.; Jiang, G. Characterization of three tetrabromobisphenol-S derivatives in mollusks from Chinese Bohai Sea: A strategy for novel brominated contaminants identification. Sci. Rep. 2015, 5, 11741.

(16) Von der Recke, R.; Vetter, W. Synthesis and characterization of 2,3-dibromopropyl-2,4,6-tribromophenyl ether (DPTE) and structur- 
ally related compounds evidenced in seal blubber and brain. Environ. Sci. Technol. 2007, 41 (5), 1590-1595.

(17) Wang, J.; Hou, Y.; Zhang, J.; Zhu, J.; Feng, Y. Transformation of 2,2',4,4'-tetrabromodiphenyl ether under UV irradiation: Potential sources of the secondary pollutants. J. Hazard. Mater. 2013, 263 (Part 2, (0)), 778-783.

(18) White, G. F.; Russell, N. J.; Tidswell, E. C. Bacterial scission of ether bonds. Microbiol. Mol. Biol. Rev. 1996, 60 (1), 216.

(19) De Jourdan, B. P.; Hanson, M. L.; Muir, D. C. G.; Solomon, K. R. Environmental fate of three novel brominated flame retardants in aquatic mesocosms. Environ. Toxicol. Chem. 2013, 32 (5), 1060-1068.

(20) Akiyama, E.; Kakutani, H.; Nakao, T.; Motomura, Y.; Takano, Y.; Sorakubo, R.; Mizuno, A.; Aozasa, O.; Tachibana, K.; Doi, T.; Ohta, S. Facilitation of adipocyte differentiation of 3T3-L1 cells by debrominated tetrabromobisphenol A compounds detected in Japanese breast milk. Environ. Res. 2015, 140, 157-164.

(21) Fujii, Y.; Nishimura, E.; Kato, Y.; Harada, K. H.; Koizumi, A.; Haraguchi, K. Dietary exposure to phenolic and methoxylated organohalogen contaminants in relation to their concentrations in breast milk and serum in Japan. Environ. Int. 2014, 63, 19-25.

(22) Cruz, R.; Cunha, S. C.; Casal, S. Brominated flame retardants and seafood safety: A review. Environ. Int. 2015, 77, 116-131.

(23) Morris, S.; Allchin, C. R.; Zegers, B. N.; Haftka, J. J.; Boon, J. P.; Belpaire, C.; Leonards, P. E.; Van Leeuwen, S. P.; De Boer, J. Distribution and fate of HBCD and TBBPA brominated flame retardants in North Sea estuaries and aquatic food webs. Environ. Sci. Technol. 2004, 38 (21), 5497-504.

(24) Koppen, R.; Becker, R.; Jung, C.; Piechotta, C.; Nehls, I. Investigation of extraction procedures and HPLC-DAD/MS for the determination of the brominated flame retardant tetrabromobisphenol A bis(2,3-dibromopropylether) in environmental samples. Anal. Bioanal. Chem. 2006, 384 (7-8), 1485-1492.

(25) Tian, Y.; Chen, J.; Ouyang, Y. Z.; Qu, G. B.; Liu, A. F.; Wang, X. M.; Liu, C. X.; Shi, J. B.; Chen, H. W.; Jiang, G. B. Reactive extractive electrospray ionization tandem mass spectrometry for sensitive detection of tetrabromobisphenol A derivatives. Anal. Chim. Acta 2014, 814, 49-54.

(26) Tian, Y.; Liu, A.; Qu, G.; Liu, C.; Chen, J.; Handberg, E.; Shi, J.; Chen, H.; Jiang, G. Silver ion post-column derivatization electrospray ionization mass spectrometry for determination of tetrabromobisphenol A derivatives in water samples. RSC Adv. 2015, 5 (23), 17474-17481.

(27) Hu, J. Y.; Jin, F.; Wan, Y.; Yang, M.; An, L. H.; An, W.; Tao, S. Trophodynamic behavior of 4-nonylphenol and nonylphenol polyethoxylate in a marine aquatic food web from Bohai Bay, north China: Comparison to DDTs. Environ. Sci. Technol. 2005, 39 (13), 48014807.

(28) Wan, Y.; Hu, J. Y.; Yang, M.; An, L. H.; An, W.; Jin, X. H.; Hattori, T.; Itoh, M. Characterization of trophic transfer for polychlorinated dibenzo-p-dioxins, dibenzofurans, non- and monoortho polychlorinated biphenyls in the marine food web of Bohai Bay, north China. Environ. Sci. Technol. 2005, 39 (8), 2417-2425.

(29) Wan, Y.; Jin, X.; Hu, J.; Jin, F. Trophic dilution of polycyclic aromatic hydrocarbons (PAHs) in a marine food web from Bohai Bay, North China. Environ. Sci. Technol. 2007, 41 (9), 3109-3114.

(30) Wang, Y.; Wang, T.; Li, A.; Fu, J.; Wang, P.; Zhang, Q.; Jiang, G. Selection of bioindicators of polybrominated diphenyl ethers, polychlorinated biphenyls, and organochlorine pesticides in mollusks in the Chinese Bohai Sea. Environ. Sci. Technol. 2008, 42 (19), 71597165.

(31) Zhu, N. L.; Li, A.; Wang, T.; Wang, P.; Qu, G. B.; Ruan, T.; Fu, J. J.; Yuan, B.; Zeng, L. X.; Wang, Y. W.; Jiang, G. B. Tris(2,3dibromopropyl) isocyanurate, hexabromocyclododecanes, and polybrominated diphenyl ethers in mollusks from Chinese Bohai Sea. Environ. Sci. Technol. 2012, 46 (13), 7174-7181.

(32) Meng, M.; Shi, J.; Liu, C.; Zhu, N.; Shao, J.; He, B.; Cai, Y.; Jiang, G. Biomagnification of mercury in mollusks from coastal areas of the Chinese Bohai Sea. RSC Adv. 2015, 5 (50), 40036-40045.
(33) Wang, X.; Liu, J.; Liu, A.; Liu, Q.; Du, X.; Jiang, G. Preparation and evaluation of mesoporous cellular foams coating of solid-phase microextraction fibers by determination of tetrabromobisphenol A, tetrabromobisphenol $\mathrm{S}$ and related compounds. Anal. Chim. Acta 2012, $753(0), 1-7$.

(34) Nyholm, J. R.; Norman, A.; Norrgren, L.; Haglund, P.; Andersson, P. L. Uptake and biotransformation of structurally diverse brominated flame retardants in zebrafish (Danio rerio) after dietary exposure. Environ. Toxicol. Chem. 2009, 28 (5), 1035-1042.

(35) Howard, P. H.; Muir, D. C. G. Identifying new persistent and bioaccumulative organics among chemicals in commerce II: Pharmaceuticals. Environ. Sci. Technol. 2011, 45 (16), 6938-6946.

(36) Kelly, B. C.; Ikonomou, M. G.; Blair, J. D.; Morin, A. E.; Gobas, F. A. Food web-specific biomagnification of persistent organic pollutants. Science 2007, 317 (5835), 236-9.

(37) Baesteros-Gómez, A.; de Boer, J.; Leonards, P. E. G. A novel brominated triazine-based flame retardant (TTBP-TAZ) in plastic consumer products and indoor dust. Environ. Sci. Technol. 2014, 48 (8), 4468-4474.

(38) Boxtel, A. L. v.; Kamstra, J. H.; Cenijn, P. H.; Pieterse, B.; Wagner, M. J.; Antink, M.; Krab, K.; Burg, B. v. d.; Marsh, G.; Brouwer, A.; Legler, J. Microarray analysis reveals a mechanism of phenolic polybrominated diphenylether toxicity in zebrafish. Environ. Sci. Technol. 2008, 42 (5), 1773-1779.

(39) Ebert, J.; B, M. Formation of PBDD/F from flame-retarded plastic materialsunder thermal stress. Environ. Int. 2003, 29, 711-716.

(40) Weber, R.; Kuch, B. Relevance of BFRs and thermal conditions on the formation pathways of brominated and brominated-chlorinated dibenzodioxins. Environ. Int. 2003, 29, 699-710.

(41) Jaeger, T.; Arsic, M.; Mayer, C. Scission of the lactyl ether eond of $\mathrm{N}$-acetylmuramic acid by Escherichia coli "etherase. J. Biol. Chem. 2005, 280, 30100-30106.

(42) Robrock, K. R.; Coelhan, M.; Sedlak, D. L.; Alvarez-Cohen, L. Aerobic biotransformation of polybrominated diphenyl ethers (PBDEs) by bacterial isolates. Environ. Sci. Technol. 2009, 43 (15), 5705-5711. 